\section{Éclairer le cerveau pour réécrire une représentation mnésique}

Stéphanie Trouche, David Dupret
MRC Brain Network Dynamics Unit,

University of Oxford, department of Pharmacology, Mansfield Road, Oxford OXI 3TH,

Royaume-Uni.

stephanie.trouche@pharm.ox.ac.uk
> La mémoire nous permet de stocker des informations liées à notre expérience passée et d'y faire appel afin d'adapter nos comportements présent et futur [1]. Garder en mémoire certains lieux est souvent facilité lorsqu'un événement saillant ${ }^{1}$ (positif ou négatif) s'y est produit $[2,3]$. Par exemple, la mémoire liée à la prise de drogue est étroitement associée au lieu dans lequel cette drogue a été consommée pouvant ainsi déclencher le besoin irrépressible de retourner dans ce lieu. Chez les mammifères, I'hippocampe a été décrit comme étant une structure cérébrale clé impliquée dans la mémoire et, plus particulièrement, la mémoire spatiale [4]. Chez le rongeur, l'hippocampe accueille des cellules dites «de lieu » qui ont la capacité de s'activer en un lieu précis de l'espace

$(\rightarrow)$ Voir le Forum de L. Rondi-Reig, $m / s$ $n^{\circ} 2$, février 2015, page 203

[5] $(\rightarrow)$.

Chaque environnement est ainsi représenté par une combinaison unique d'activité de cellules de lieu permettant au cerveau de construire une représentation neuronale interne de cet environnement [6]. Dans notre étude, nous avons testé si, en inactivant spécifiquement la représentation spatiale d'un environnement préalablement associé à l'expérience d'une prise de drogue telle que la cocaïne, il était possible de rectifier certains comportements indésirables tels que la recherche active de drogue dans ce même environnement [7]. Les méthodes conventionnelles, comme les lésions pharmacologiques

\footnotetext{
${ }^{1}$ Vif, frappant.
}

et/ou chirurgicales, ne sont pas adaptées pour répondre à cette question car elles touchent une large population de neurones. Or, la représentation spatiale liée à l'expérience d'une drogue correspond à un réseau neuronal local, intriqué avec d'autres réseaux correspondant à d'autres représentations. Les méthodes de lésion ne permettent donc pas de cibler uniquement la représentation choisie et affectent d'autres représentations importantes à conserver. Une nouvelle technique visant une seule représentation a ainsi été développée.

Inactiver les neurones porteurs d'une représentation spatiale

Afin d'inhiber sélectivement les neurones de l'hippocampe participant à la représentation spatiale d'un environnement donné, nous avons développé une stratégie combinant l'utilisation d'une souris transgénique, appelée TetTag [7-9], avec une approche optogénétique [10]. La souris TetTag exprime un transgène contenant l'activateur de transcription tétracycline ( $\mathrm{tTa}$ ) sous le contrôle du promoteur $c$-fos. Nous avons injecté cette souris avec un virus exprimant ArchT-GFP (Archaerhodopsin-green fluorescent protein) sous le contrôle du promoteur TRE3G (tetracyclin responsive element $3 G$ ), ciblant I'hippocampe. Cette stratégie permet de faire exprimer, uniquement dans les neurones de l'hippocampe qui étaient recrutés lors de l'exploration d'un environnement donné, l'« inactivateur neuronal » ArchT, une pompe à protons sensible à la lumière [11]. Ces neurones «étiquetés » par ArchT sont ainsi sensibles à la lumière: une illumination locale permet de bloquer la réponse neuronale. Cet étiquetage sélectif est contrôlé par la présence de l'antibiotique doxycycline (Dox) dans la nourriture des souris. En absence de Dox, la tTa se lie au promoteur TRE3G, induisant l'expression de ArchT-GFP dans les neurones exprimant c-fos. c-fos étant un marqueur de l'activité neuronale, seuls les neurones répondant à l'environnement auquel la souris est exposée sont donc étiquetés par ArchT. Lorsque les animaux sont nourris avec la Dox, l'activation de la tTA est bloquée et les neurones ne peuvent pas/ plus exprimer ArchT. Cet outil nous a permis, pour la première fois, de rendre les neurones de l'hippocampe représentant un environnement donné transitoirement silencieux à chaque épisode de lumière.

\section{Inactiver les neurones étiquetés} entraîne le recrutement des neurones alternatifs

Nous avons tout d'abord examiné les conséquences de la photo-inhibition ${ }^{2}$ des neurones étiquetés sur l'activité générale de l'hippocampe. L'optogénétique rend possible le contrôle de l'activité des circuits neuronaux avec une grande précision spatio-temporelle, en illuminant ces neurones par épi$(\rightarrow)$ Voir la Synthèse de G.P. Dugué et L. Tricoire, $m / s n^{\circ} 3$, mars 2015, page 291 sodes brefs [12] $(\rightarrow)$.

Dans notre étude, nous avons implanté des fibres optiques ainsi que des électrodes d'enregistrements ciblant l'hip-

${ }^{2}$ Inactivation par illumination dans le cadre d'une procédure d'optogénétique. 


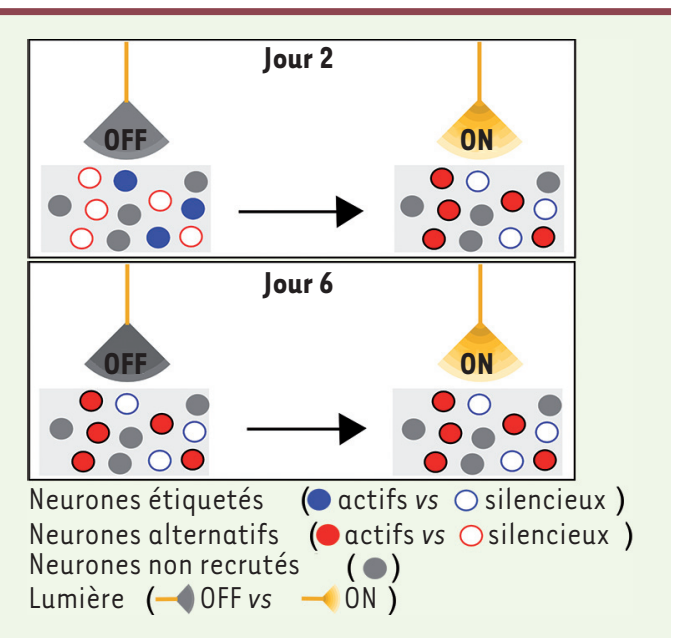

Figure 1. Émergence d'une représentation spatiale alternative. Les neurones de l'hippocampe de souris ont été enregistrés pendant l'exploration d'un environnement spatial. Au $2^{\mathrm{e}}$ jour d'enregistrement, les neurones étiquetés (c'est-à-dire actifs lors de l'exploration de l'environnement le $1^{\text {er }}$ jour et exprimant ArchT [Archaerhodopsin]) étaient activés pendant l'exploration de l'environnement (lumière OFF, ronds bleus pleins), participant ainsi à sa représen-

tation spatiale. Ils sont devenus silencieux lors des épisodes de photo-inhibition (lumière ON, ronds bleus vides). Ce phénomène a entraîné l'activation d'une seconde population de neurones voisins (dits alternatifs, ronds rouges pleins), qui étaient initialement silencieux (ronds rouges vides). La photo-inhibition répétée des neurones étiquetés les jours qui ont suivi a empêché ces neurones étiquetés de s'activer lors de l'exploration (lumière ON ou OFF). Les neurones alternatifs ont cependant pris le relais en maintenant leur activation, offrant ainsi une représentation alternative de cet environnement.

pocampe, afin d'inactiver les neurones étiquetés (exprimant ArchT et correspondant à la représentation de l'environnement précédemment exploré) et enregistrer les conséquences sur l'activité des neurones de l'hippocampe pendant que les souris ré-exploraient l'environnement (Figure 1). Alors que notre intervention visait à spécifiquement inhiber les neurones étiquetés, nous avons été surpris d'observer l'émergence d'une seconde population de neurones dits «alternatifs » présentant, au contraire, une activité accrue pendant la photo-inhibition.

L'émergence d'une seconde population de neurones fournit une carte spatiale alternative

Quelles sont les caractéristiques fonctionnelles qui différencient ces deux populations de neurones? Lors de la réexposition des animaux à l'environnement (sans photo-inhibition), les neurones étiquetés lors de la première exposition ont été recrutés et présentaient des caractéristiques de cellules de lieu, c'est-à-dire qu'ils émettaient des potentiels d'action à des positions spécifiques de l'environnement. Ce n'était pas le cas des neurones alternatifs qui étaient dans un état silencieux. $\varepsilon n$ revanche, après des épisodes répétés de photo-inhibition de l'hippocampe, les neurones étiquetés ont été inactivés de façon durable et ont par conséquent perdu leur caractéristique de cellules de lieu. Cette procédure a par ailleurs « réveillé » d'autres neurones voisins (les neurones alternatifs) et a révélé leur propriété de cellules de lieu. Notre intervention optogénétique a donc permis un échange de rôles: en désengageant le recrutement initial de neurones dans la représentation spatiale d'un environnement donné, elle a autorisé d'autres neurones à prendre le relais et à fournir une représentation spatiale alternative de ce même environnement (Figure 1).

\section{Réécrire une représentation mnésique pour réajuster un comportement}

$\varepsilon n$ substituant une représentation spatiale par une autre, est-il possible de modifier un comportement indésirable?
Plus précisément, le lien mnésique rattachant un lieu à l'expérience de drogue peut-il être rompu et ainsi empêcher la recherche active de drogue? Les souris sont capables de mémoriser un environnement lié à l'expérience de la prise de cocaïne comme cela est mis en évidence par le temps accru qu'elles passent dans cet environnement par rapport à un environnement à valence ${ }^{3}$ neutre [7]. En utilisant la stratégie optogénétique décrite précédemment, nous avons étiqueté avec ArchT les neurones de l'hippocampe recrutés pendant l'exploration de l'environnement dans lequel la cocaïne a été consommée. Les jours suivants, les souris ont été réexposées dans ce même environnement, mais sans la présence de cocaïne, et les neurones étiquetés ont été photo-inhibés. Cette photo-inhibition répétée, en faisant émerger une nouvelle représentation spatiale portée par les neurones alternatifs devenus actifs, a ensuite empêché les souris de retourner dans l'environnement associé à la cocaïne.

\section{Conclusion}

Notre étude révèle qu'il est possible de réécrire une représentation mnésique spatiale liée à l'expérience d'une drogue et ce, en éteignant l'activité des neurones engagés dans la représentation de cette mémoire causant ainsi un autre groupe de neurones à prendre le relais. Cette approche, fondée sur l'une des fonctions essentielles de l'hippocampe - la représentation d'environnements pourrait potentiellement être utilisée pour atténuer les symptômes de peur persistants, évoqués lors de la réexposition au lieu dans lequel des individus ont vécu une expérience traumatisante. $\diamond$ Illuminating the brain to rewrite a memory representation

\section{REMERCIEMENTS}

Nous remercions vivement tous les co-auteurs de cette étude, les membres de l'équipe, ainsi que les

\footnotetext{
${ }^{3}$ Une valence positive (respectivement négative) correspond à une valeur agréable (respectivement désagréable) ou une émotion positive (respectivement négative).
} 
membres de l'Unité Brain Network Dynamics (BNDU, $M R C$, Université d'Oxford). Ce travail a été financé par le Medical Research Council UK et le Medical Research Foundation.

\section{LIENS D'INTÉRÊT}

Les auteurs déclarent n'avoir aucun lien d'intérêt concernant les données publiées dans cet article.

\section{RÉFÉRENCES}

1. Schacter DL, Addis DR, Buckner RL. Remembering the past to imagine the future: the prospective brain. Nat Rev Neurosci $2007 ; 8$ : 657-61.
2. Ramirez $S$, Liu $X$, Lin PA, et al. Creating a false memory in the hippocampus. Science 2013 ; 341 : 387-91.

3. Redondo RL, Kim J, Arons AL, et al. Bidirectional switch of the valence associated with a hippocampal contextual memory engram. Nature $2014 ; 513$ : 426-30

4. O'Keefe J, Nadel L. The hippocampus as a cognitive map. Oxford: Clarendon Press, 1978 : 570 p.

5. Rondi-Reig L. Le codage de l'espace : journal de bord d'un prix Nobel. Med Sci (Paris) 2015 ; 31 : 203-8.

6. O'Keefe J, Dostrovsky J. The hippocampus as a spatial map. Preliminary evidence from unit activity in the freely-moving rat. Brain Res $1971 ; 34: 171-5$.

7. Trouche S, Perestenko PV, van de Ven GM, et al. Recoding a cocaine-place memory engram to a neutral engram in the hippocampus. Nat Neurosci $2016 ; 19: 564-7$.
8. Reijmers LG, Perkins BL, Matsuo N, Mayford M. Localization of a stable neural correlate of associative memory. Science 2007 ; 317 : 1230-3.

9. Trouche S, Sasaki JM, Tu T, Reijmers LG. Fear extinction causes target-specific remodeling of perisomatic inhibitory synapses. Neuron $2013 ; 80$ : 1054-65.

10. Boyden ES. Optogenetics and the future of neuroscience. Nat Neurosci $2015 ; 18: 1200-1$.

11. Han X, Chow BY, Zhou H, et al. A high-light sensitivity optical neural silencer: development and application to optogenetic control of non-human primate cortex. Front Syst Neurosci $2011 ; 5: 18$.

12. Dugué GP, Tricoire L. Principes et applications de I'optogénétique en neuroscience. Med Sci (Paris) $2015 ; 31: 291-303$.
NOUVELLE

\section{Un mécanisme d'activation cystéine-dépendant pour les ligands pro-inflammatoires de RAGE?}

Laure Yatime
Laboratoire de dynamique des interactions membranaires normales et pathologiques (DIMNP) - UMR5235, CNRS, Inserm, université de Montpellier, place Eugène Bataillon, bâtiment 24 cc.107, 34095, Montpellier Cedex 5, France. laure.yatime@inserm.fr

incluant la protéine HMGBI (high mobility group box 1), plusieurs membres de la famille des protéines $S 100^{l}$ ou encore les peptides amyloïdes $\beta$ [3]. Le trait commun à tous ces ligands est que leur présence dans le fluide extracellulaire est conditionnée par la pathologie qui sous-tend leur formation et/ou leur surexpression (cancers pour les protéines $S 100$, septicémie ou cancer pour HMGBl, maladies neurodégénératives pour les peptides amyloïdes $\beta$, diabète pour les $A G \varepsilon$ ). RAGE est ainsi capable de détecter des signaux de danger multiples associés à des pathologies humaines très variées [3].

RAGE, le « Dr Jekyll et M. Hyde » de l'inflammation

La reconnaissance de ces DAMP par RAGE enclenche des cascades de signalisa-

\footnotetext{
${ }^{1}$ Les protéines $\mathrm{S100}$, qui forment une famille, sont associées à un grand nombre de pathologies infectieuses ou inflammatoires.
}

\section{spécialisé dans la reconnaissance}

\section{de dangers endogenes}

une machinerie complexe et diversifiée pour détecter le non-soi et le soi altéré qui permettra leur élimination de notre organisme et le retour à l'homeostasie. des récepteurs spécifiques appelés PRR (pattern recognition receptors) capables gène (PAMP pour pathogen-associated molecular patterns)

ou endogène (DAMP

pour damage-asso-

ciated molecular

de J. Claverie et al.,

$m / s n^{\circ} 4$, avril 2016,

page 335

Parmi ces PRR, RAGE (receptor for advanced glycation end-products) est un récepteur transmembranaire exprimé à la surface de nos cellules qui reconnaît des molécules de danger exclusivement endogènes (DAMP) libérees dans le milieu extracellulaire dans à la découverte de RAGE, au début des années 1990, l'ont identifié comme le products) [2]. Les AGE corresp à des protéines cette modification $m / s n^{\circ} 2$, février 2017 page 176

l'altération des fonctions survient irréversiblem forcé par la présence d'un fort taux de glucose dans le sang. II favorise ainsi l'apparition de complications sévères, notamment chez les personnes atteintes de la maladie d'Alzheimer ou chez les diabétiques. Au cours des vingt dernières années, un large répertoire de ligands de RAGE a été caractérisé 